\title{
Constrained stabilization problems for discrete-time linear plants
}

\author{
Ali Saberi $^{1} \quad$ Jian Han ${ }^{1} \quad$ Anton A. Stoorvogel ${ }^{2,3} \quad$ Guoyong Shi $^{1}$ \\ ${ }^{1}$ School of Electrical Engineering and Computer Science, Washington State University, \\ Pullman, WA 99164-2752, U.S.A., \{saberi,jhan,gshi\} @eecs.wsu.edu \\ 2 Department of Mathematics and Computing Science, Eindhoven Univ. of Technology, P.O. Box 513, \\ 5600 MB Eindhoven, The Netherlands, A.A.Stoorvogel@tue.nl \\ ${ }^{3}$ Department of Information Technology and Systems, Delft University of Technology, P.O. Box 5031, \\ 2600 GA Delft, The Netherlands, A.A.Stoorvogel@its.tudelft.nl
}

\begin{abstract}
In this paper we discuss discrete-time systems which have constraints on all or part of the inputs as well as on the states of the system. We show that solvability of stabilization problems is closely related to two important concepts: right-invertibility of the constraints and the constraint invariant zeros. In the case of right-invertible constraints we give constructive methods to determine suitable controllers.
\end{abstract}

\section{Introduction}

Two most commonly encountered constraints in control engineering are actuator constraints and state constraints. References [1] and [8] capture some recent research activities regarding constraints on actuators, i.e. on inputs. Besides the actuator constraints, state constraints are a major concern as well in control engineering. However, state constraints, unlike actuator constraints, have not received much attention from a structural point of view. There have been some efforts on dealing with state and input constraints utilizing the concept of positive invariant sets [2] and techniques of model predictive control $[3,4]$. However, the available tools along these lines are computationally very demanding and the resulting controllers are highly complicated.

During the last decade several aspects of control design problems for linear systems with magnitude and rate constraints on control variables have been studied among others by the first and third author and their students and collaborators. A number of powerful analysis and design methods such as low gain, low-high gain, scheduled low gain, scheduled low-high gain and many variations of them have been developed for several core control design problems including global and semi-global internal stabilization, external stabilization, output regulation, and disturbance rejection. Having thus studied the problems associated with constraints on control variables, the research thrust of the first and third author and their students has broadened to include magnitude and rate constraints on control variables as well as state variables. In connection with stabilization, whenever amplitude and rate constraints on both state as well as input variables exist, a taxonomy of all possible constraints is introduced, and several fundamental results on global, semi- global, and regional stabilization are developed in a recent paper [5]. The work of [5] focuses on continuous-time systems and generalizes, extends, and covers all existing results including those developed in [7]. The focus of this paper is on discrete-time systems.

It is becoming evident that the taxonomy of constraints developed in [5] plays dominant roles in every type of control design problem not only for continuous-time systems but also for discrete-time systems. The taxonomy of constraints is developed by appropriately modeling the constraints in terms of what is called a constraint output (of the given system) which is specified to lie in a prescribed constraint set. It turns out that the mapping from the input to the constraint output vector or more specifically its structural properties play dominant roles in dictating what is feasible and what is not feasible. Based on their impact for control purposes, such structural properties have been categorized in two directions both of which have profound impact on what can or cannot be achieved for a given system. One direction of categorization is based on the right invertibility or lack of it of the mapping from the input to the constraint output vector. This direction of categorization delineates the constraints into two mutually exclusive categories, (1) right invertible constraints representing the case when the mapping from the input to the constraint output vector is right invertible, and (2) non-right invertible constraints representing the case when the mapping from the input to the constraint output vector is not right invertible. Another direction of categorization is based on so called constraint invariant zeros of the plant, i.e. the invariant zeros of the mapping from the input to the constraint output vector. Like in the case of right-invertibility or lack of it, this direction of categorization delineates the constraints into two main mutually exclusive categories, (1) at most weakly non-minimum phase constraints representing the case when the constraint invariant zeros are in the closed left half complex plane for continuous-time systems or in the closed unit disc for discrete-time systems, and (2) strongly non-minimum phase constraints representing the case when one or more of the constraint invariant zeros are in the open right half complex plane for continuous-time systems or outside the unit 
disc for discrete-time systems. Based on such a taxonomy of constraints, two main features emerge:

- Neither the constrained semi-global nor the constrained global stabilization problem is solvable whenever the constraints are strongly non-minimum phase.

- There exists a perceptible demarkation line between the right and non-right invertible constraints. In particular, the solvability conditions for the constrained semi-global and global stabilization problems do not depend on the shape of the constraint set(s) for right invertible constraints whereas for non-right invertible constraints they indeed do so.

This paper which dwells with discrete-time systems focuses on the above aspects and draws contrast with continuoustime systems. Although the development for discrete-time systems parallels somewhat that in continuous-time systems, there are several fundamental differences between continuous- and discrete-time systems: (1) the solvability conditions for the posed problems differ from those of continuous-time systems; for instance, unlike in continuoustime systems, for semi-global stabilization, the order of the infinite zeros of a discrete-time system cannot be greater than two, (2) techniques of proofs are different, and (3) the methods of constructing appropriate controllers need to be revised as well.

\section{Problem formulation}

Consider a discrete-time linear system:

$$
\Sigma_{d}:\left\{\begin{aligned}
x(k+1) & =A x(k)+B u(k) \\
y(k) & =C_{y} x(k)+D_{y} u(k) \\
z(k) & =C_{z} x(k)+D_{z} u(k)
\end{aligned}\right.
$$

where $x \in \mathbb{R}^{n}, u \in \mathbb{R}^{m}, y \in \mathbb{R}^{r}, z \in \mathbb{R}^{p}$ are respectively state, input, measurement output and constrained output as shown in the following figure:

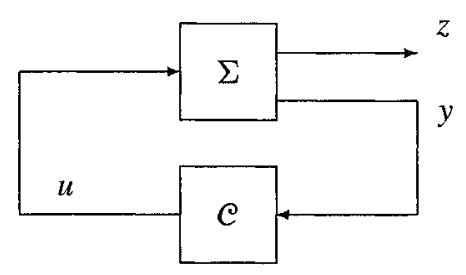

For two a priori given sets $s \subset \mathbb{R}^{p}$ and $\mathcal{T} \subset \mathbb{R}^{p}$, we are interested in stabilization of the plant $\Sigma$ subject to the constraint that the output $z(k)$ remains in the set $\delta$ for all $k \geqslant 0$ while the variation rate of $z(k)$, i.e. $z(k+1)-z(k)$, remains in the set $\mathcal{T}$. In particular, we are interested in obtaining necessary and sufficient conditions for the existence of such a feedback. We will also present design methodologies to achieve constrained stabilization.
We make the following fundamental assumption on the nature of the constraint sets $\&$ and $\mathcal{T}$ :

Assumption 2.1 The following conditions on $\&$ and $\mathcal{T}$ are satisfied:

(i) The sets $\&$ and $\mathcal{T}$ are closed, convex and contain 0 as an interior point.

(ii) $\& \cap \mathcal{T}$ is bounded.

(iii) We have $C_{z}^{T} D_{z}=0$ and

$$
\begin{aligned}
& \&=\left(\& \cap \operatorname{im} C_{z}\right)+\left(\& \cap \operatorname{im} D_{z}\right) \\
& \mathcal{T}=\left(\mathcal{T} \cap \operatorname{im} C_{z}\right)+\left(\mathcal{T} \cap \operatorname{im} D_{z}\right)
\end{aligned}
$$

Remark. We observe that im $C_{z}$ reflects the state constraints while im $D_{z}$ reflects the input constraints. Therefore the decomposition of $\&$ and $\mathcal{T}$ as required in (iii) only implies that we have constraints on states and/or inputs.

The following notion is necessary before we proceed to the problem formulation.

Definition 2.2 Let the system (2.1) and constraint sets \& and $\mathcal{T}$ be given. We define

$$
\begin{array}{r}
\mathcal{V}(\delta, \mathcal{T}):=\left\{x_{0} \in \mathbb{R}^{p} \mid \exists u_{0} \text { such that } C_{z} x_{0}+D_{z} u_{0} \in \mathcal{S}\right. \\
\text { and } \left.C_{z}\left(A x_{0}+B u_{0}\right)-C_{z} x_{0} \in \mathcal{T}\right\}
\end{array}
$$

as the admissible set of initial conditions.

We formulate our problems either in global or in semi-global setting.

Problem 2.3 Semi-global constrained stabilization via state feedback Consider the system (2.1) along with constrained sets $\& \subset \mathbb{R}^{p}$ and $\tau \subset \mathbb{R}^{p}$. For any a priori given bounded set $\mathcal{W}$ contained in the interior of $\mathcal{V}(\delta, \mathcal{T})$ find, if possible, a state feedback (possibly nonlinear) $u=f(x, k)$ such that the following conditions hold:

(i) The equilibrium point $x=0$ of the closed-loop system is asymptotically stable with $\mathcal{W}$ contained in its basin of attraction.

(ii) For any $x_{0} \in \mathcal{W}, z(k) \in \delta$ and $z(k+1)-z(k) \in \mathcal{T}$ for all $k>0$.

Problem 2.4 (Global constrained stabilization via state feedback) Consider the system (2.1) along with constrained sets $\delta \subset \mathbb{R}^{p}$ and $\mathcal{T} \subset \mathbb{R}^{p}$. Find, if possible, a state feedback (possibly nonlinear) $u=f(x, k)$ such that the following conditions hold:

(i) The equilibrium point $x=0$ of the closed-loop system is asymptotically stable with $\mathcal{V}(\delta, \mathcal{T})$ contained in its basin of attraction. 
(ii) For any $x_{0} \in \mathcal{V}(\&, \mathcal{T}), z(k) \in \&$ and $z(k+1)-z(k) \in$ $\tau$ for all $k>0$.

Problem 2.5 (Semi-global constrained stabilization via measurement) Consider a system (2.1) along with constraint sets $\& \subset \mathbb{R}^{p}$ and $\mathcal{T} \subset \mathbb{R}^{p}$. For any a priori given bounded set $W$ contained in the interior of the set $\mathcal{V}(\mathcal{S}, \mathcal{T}) \times \mathbb{R}^{s}$, find, if possible, a measurement feedback (possibly nonlinear and time-varying) of the form,

$$
\left\{\begin{aligned}
p(k+1) & =l(p(k), y(k), k), \quad p \in \mathbb{R}^{s} \\
u(k) & =g(p(k), y(k), k)
\end{aligned}\right.
$$

such that the following conditions hold:

(i) The equilibrium point $(x, p)=(0,0)$ of the closedloop system is asymptotically stable with $W$ contained in its basin of attraction.

(ii) For any $\left(x_{0}, p_{0}\right) \in \mathcal{W}$, we have $z(k) \in \delta$ and $z(k+1)-z(k) \in \mathcal{T}$ for all $k>0$.

Remark. It is natural to formulate a global constrained stabilization problem via measurement feedback along the same lines as in the above problems. However, we have not done so here mainly because, except for some very special cases, a global constrained stabilization problem via measurement feedback is not solvable. See [5] for a discussion.

\section{Taxonomy of constraints}

We let $\mathbb{C}, \mathbb{C}^{\oplus}, \mathbb{C}^{\ominus}$ and $\mathbb{C}^{0}$ denote respectively the set of complex numbers in the entire complex plane, outside the unit circle, inside the unit circle, and on the unit circle.

The following notions are fundamental to the taxonomy of constraints given below.

Definition 3.1 The system:

$$
\left\{\begin{aligned}
x(k+1) & =A x(k)+B u(k) \\
y(k) & =C x(k)+D u(k)
\end{aligned}\right.
$$

where $x \in \mathbb{R}^{n}, u \in \mathbb{R}^{m}$ and $y \in \mathbb{R}^{p}$, is said to be right invertible if, for any $y_{\text {ref }}(k)$ defined on $[0, \infty)$, there exists $a u(k)$ and a choice of $x(0)$ such that $y(k)=y_{\text {ref }}(k)$ for all $k \in[0, \infty)$.

Definition 3.2 The invariant zeros of a linear system with a realization $(A, B, C, D)$ are those points $\lambda \in \mathbb{C}$ for which

$$
\operatorname{rank}\left(\begin{array}{cc}
\lambda I-A & -B \\
C & D
\end{array}\right)<\operatorname{normrank}\left(\begin{array}{cc}
s I-A & -B \\
C & D
\end{array}\right)
$$

where "normrank" denotes normal rank.
The first categorization is based on whether the subsystem from $u$ to $z$ is right invertible or not. The constraints are said to be

- right invertible constraints if the subsystem $\Sigma_{z}$ : $\left(A, B, C_{z}, D_{z}\right)$ is right invertible.

- non-right invertible constraints if the subsystem $\Sigma_{z}$ : $\left(A, B, C_{z}, D_{z}\right)$ is not right invertible.

It turns out that the location of the invariant zeros of the subsystem from $u$ to $z$ is also important in characterizing the solvability of stabilization problems. We refer to these invariant zeros as constraint invariant zeros:

Definition 3.3 The invariant zeros of the system characterized by the quadruple $\left(A, B, C_{z}, D_{z}\right)$ are called constraint invariant zeros of the given system $\Sigma$.

The second categorization of constraints is based on the location of the constraint invariant zeros. The constraints are said to be

- minimum phase constraints if all the constraint invariant zeros are in $\mathbb{C}^{\ominus}$.

- weakly minimum phase constraints if all the constraint invariant zeros are in $\mathbb{C}^{\ominus} \cup \mathbb{C}^{0}$ with the restriction that any invariant zero in $\mathbb{C}^{0}$ is simple,

- weakly non-minimum phase constraints if all the constraint invariant zeros are in $\mathbb{C}^{\ominus} \cup \mathbb{C}^{0}$ with at least one non-simple invariant zero in $\mathbb{C}^{0}$.

- at most weakly non-minimum phase constraints if all the constraint invariant zeros are in $\mathbb{C}^{\ominus} \cup \mathbb{C}^{0}$.

- strongly non-minimum phase constraints if at least one constraint invariant zeros is in $\mathbb{C}^{\oplus}$.

\section{Right invertible constraints}

In this section we provide necessary and sufficient conditions for the solvability of Problems 2.3 and 2.4, under the assumption that the subsystem $\left(A, B, C_{z}, D_{z}\right)$ is right invertible, i.e. the right invertible constraints. In Section 6 we concentrate on the case where $\left(A, B, C_{z}, D_{z}\right)$ is non-right invertible and clarify the intrinsic additional difficulties we encounter in the general case.

Theorem 4.1 Consider the plant $\Sigma_{d}$ as given by (2.1). Assume that the system characterized by $\left(A, B, C_{z}, D_{z}\right)$ is right-invertible. Let the sets $\&$ and $\mathcal{T}$ satisfy assumption 2.1. Moreover, assume the set $\&$ is bounded. Then the global or semi-global constrained stabilization problem as defined in Problem 2.3 or Problem 2.4 is solvable if and only if:

(i) $(A, B)$ is stabilizable. 
(ii) The constraint invariant zeros of the given system $\Sigma_{d}$ are all in the closed unit disc, i.e. the system $\Sigma_{d}$ has at most weakly non-minimum phase constraints.

(iii) The subsystem $\left(A, B, C_{z}, D_{z}\right)$ has no infinite zeros of order greater than one.

Proof : The main part of the proof is to design an appropriate control law. The construction of such a control law is outlined in the next section.

For the case of measurement feedback, we have the following theorem which, due to space limitations, we will not prove in this paper.

Theorem 4.2 Consider the plant $\Sigma_{d}$ as given by (2.1). Assume that the system characterized by $\left(A, B, C_{z}, D_{z}\right)$ is right invertible. Let the sets $\&$ and $\mathcal{T}$ satisfy Assumption 2.1 . Then the semi-global constrained stabilization problem via measurement feedback as defined in Problem 2.5 is solvable if the following conditions hold:

(i) $(A, B)$ is stabilizable.

(ii) The constraint invariant zeros of the given system $\Sigma$ are all in the closed unit disc, i.e. the system $\Sigma$ has at most weakly non-minimum phase constraints.

(iii) The subsystem $\left(A, B, C_{z}, D_{z}\right)$ has no infinite zeros of order greater than one.

(iv) The pair $\left(C_{y}, A\right)$ is observable.

(v) $\operatorname{ker} C_{z} \subset \operatorname{ker} C_{z} A$.

(vi) $\operatorname{ker}\left(\begin{array}{ll}C_{y} & D_{y}\end{array}\right) \subset \operatorname{ker}\left(\begin{array}{ll}C_{z} & D_{z}\end{array}\right)$

Moreover, conditions ( $i$ ) and (ii) are necessary for the solvability of the semi-global constrained stabilization problem via measurement feedback as defined in Problem 2.5.

Note that condition (vi) states that the constrained output is part of the measurements.

\section{Controller design}

The design of control laws for constrained stabilization will largely rely on the structure of the underlying system. For this aim, the original system needs to be rewritten in a special coordinate basis so that the system properties involving invariant zeros and infinite zeros are revealed naturally. A detailed special coordinate basis (scb) is presented in [6]. In terms of scb, there exist a set of three coordinate bases, one in input space, another in state space, and yet another in output space where $z$ lies, and an output injection matrix $K_{1}$ such that the subsystem $\Sigma_{z u}\left(A, B, C_{z}, D_{z}\right)$ can be rewritten as (we slightly abuse the notation of scb given in [6] in order to keep the system variables easily tractable):

$$
\begin{aligned}
&\left(\begin{array}{l}
x_{1}(k+1) \\
x_{2}(k+1)
\end{array}\right)=\left(\begin{array}{cc}
A_{11} & 0 \\
A_{21} & A_{22}
\end{array}\right)\left(\begin{array}{c}
x_{1}(k) \\
x_{2}(k)
\end{array}\right)+\left(\begin{array}{c}
0 \\
B_{2}
\end{array}\right) u(k) \\
&+\left(\begin{array}{c}
K_{1} \\
0
\end{array}\right) z(k) \\
& y(k)=\left(\begin{array}{ll}
C_{y, 1} & C_{y, 2}
\end{array}\right)\left(\begin{array}{l}
x_{1}(k) \\
x_{2}(k)
\end{array}\right)+D_{y} u(k) \\
& z(k)=\left(\begin{array}{ll}
C_{z, 1} & C_{z, 2}
\end{array}\right)\left(\begin{array}{l}
x_{1}(k) \\
x_{2}(k)
\end{array}\right)+D_{z} u(k)
\end{aligned}
$$

where $x_{1} \in \mathbb{R}^{n_{1}}$ and $x_{2} \in \mathbb{R}^{n_{2}}$. This decomposition renders the subsystem characterized by the quadruple $\left(A_{22}, B_{2}\right.$, $\left.C_{z, 2}, D_{z}\right)$ strongly controllable and having no finite zeros (see [6]).

In the rest of this section, we assume that the constraints are right invertible. In this case we have some additional structure in the sense that we have $C_{z, 1}=0$ and therefore the $z$-equation in (5.1) becomes

$$
z=\left(\begin{array}{ll}
0 & C_{z, 2}
\end{array}\right)\left(\begin{array}{l}
x_{1} \\
x_{2}
\end{array}\right)+D_{z} u .
$$

The decomposition above reveals that the controller design can be accomplished in two layers. Moreover, we can characterize the domain of attraction of the original system in terms of two subsystems. To this end, we first need a concept.

Definition 5.1 Consider the system:

$$
\bar{\Sigma}:\{x(k+1)=A x(k)+B u(k)
$$

where $u(k) \in s_{0}$ and $u(k+1)-u(k) \in \mathcal{T}_{0}$, where $s_{0}$ and $\mathcal{T}_{0}$ are convex sets which contain zero as an interior point and are such that $s_{0} \cap \mathcal{T}_{0}$ is bounded. We define the region $\mathcal{R}_{c}\left(\vec{\Sigma}, \varsigma_{0}, \mathcal{J}_{0}\right)$ of asymptotic null-controllability with input constraint sets $\delta_{0}$ and $\mathcal{I}_{0}$ to be the set of initial conditions $x_{0}$ such that there exists $u(k)$ satisfying $u(k) \in \&$ and $u(k+1)-u(k) \in \mathcal{T}$ for all $k>0$ and $\lim _{k \rightarrow \infty} x(k)=0$ for all $x(0)=x_{0}$.

The zero dynamics of the subsystem characterized by $(A, B$, $C_{z}, D_{z}$ ) are the dynamics of $x_{1}$ in (5.1) and therefore given by:

$$
\Sigma_{s}: x_{1}(k+1)=A_{11} x_{1}(k)+K_{1} z(k)
$$

which, by viewing $z$ as input, has input constraints in the sense that we must have that $z(k) \in \&$ and $z(k+1)-z(k) \in$ $\mathcal{T}$ for all $k>0$. Define $\mathcal{R}_{C}\left(\Sigma_{s}, \&, \mathcal{T}\right)$ as the region of asymptotic null-controllability of system $\Sigma_{s}$ with input constraint sets $\&$ and $\mathcal{T}$.

Next consider the subsystem:

$$
\left\{\begin{aligned}
x_{2}(k+1) & =A_{22} x_{2}(k)+B_{2} u(k)+A_{21} x_{1}(k) \\
z(k) & =C_{z, 2} x_{2}(k)+D_{z} u(k)
\end{aligned}\right.
$$


which is extracted from system (5.1). We define:

$$
\begin{gathered}
\mathcal{V}_{2}(\mathcal{S}, \mathcal{T})=\left\{x_{2,0} \in \mathbb{R}^{p_{2}} \mid \exists u_{0}\right. \text { such that } \\
C_{z, 2} x_{2,0}+D_{z} u_{0} \in \mathcal{S} \text { and } \\
C_{z, 2}\left(A x_{2,0}+B_{2} u_{0}\right)-C_{z, 2} x_{2,0} \in \mathcal{T} \\
\text { where } \left.x_{2,0}=x_{2}(0)\right\}
\end{gathered}
$$

Then we have the following theorem:

Theorem 5.2 Consider the plant $\Sigma_{d}$ as given by (2.1). Assume $\mathcal{T}=\mathbb{R}^{p}$ (i.e. no rate constraints are present). Moreover, let the subsystem from $u$ to $z$ be right invertible and assume the constraint set \& satisfies Assumption 2.1. For a given stabilizing controller $u=f(x)$, denote its domain of stabilization as $\mathcal{R}_{A}^{f}(\Sigma)$. Then we have:

$$
\mathcal{R}_{A}^{f}(\Sigma) \subseteq \mathcal{R}_{C}\left(\Sigma_{s}, \&, \mathbb{R}^{p}\right) \times \mathcal{V}_{2}\left(\&, \mathbb{R}^{p}\right)
$$

Moreover, if $z=g\left(x_{1}\right)$ is a stabilizing controller for the subsystem (5.3) such that $z \in \&$ with domain of stabilization $\mathcal{R}_{A}^{g}\left(\Sigma_{s}\right)$, then we can find for any compact set $\mathcal{R}$, satisfying $\mathcal{R} \subset \rho \mathcal{R}_{A}^{g}\left(\Sigma_{s}\right)$ for some $\rho<1$, a stabilizing controller $u=g(x)$ for the complete system $\Sigma_{d}$ whose domain of stabilization contains $\mathcal{R} \times \mathcal{V}_{2}\left(\&, \mathbb{R}^{p}\right)$ and such that the closed loop system satisfies the constraints $z \in \&$ for all $k \geqslant 0$ and for all initial conditions in $\mathcal{R} \times \mathcal{V}_{2}\left(\&, \mathbb{R}^{p}\right)$.

Remark. A fundamental aspect of solvability conditions as given by Theorems 4.1 and 4.2 is that they are independent of any specific features of the given constraint sets. That is, for the case of a right invertible system $\Sigma_{d}$, if the semi-global or global constrained stabilization problems are solvable for some given constraint sets satisfying Assumption 2.1, then these problems are also solvable for all constraint sets satisfying Assumption 2.1.

Due to space limitations, we only present the main steps of controller design. The details are omitted.

Step 1: We first choose a feedback for $z$ such that the zero dynamics is globally or semiglobally stable. More specifically, given $\varepsilon$ and $\delta$, we choose $z_{0}=f\left(x_{1}\right)$ such that for the system:

$$
x_{1}(k+1)=A_{11} x_{1}(k)+K_{1} z_{0}(k)+K_{1} v(k)
$$

we have:

- (5.6) is stable in the sense that for all initial conditions (global case) or all initial conditions in some arbitrarily large but compact subset $\mathcal{W}_{1}$ (semiglobal case) and any disturbance $v$ satisfying

$$
\|v(k)\| \leqslant M \lambda^{k}
$$

we have that $x_{1}(k) \rightarrow 0$ as $k \rightarrow \infty$

- We have for all $k$ :

$$
\begin{gathered}
f\left(x_{1}(k)\right) \in \delta, \quad f\left(x_{1}(k+1)\right)-f\left(x_{1}(k)\right) \in \mathcal{T}, \\
\left\|f\left(x_{1}(k)\right)\right\|<\varepsilon, \quad\left\|f\left(x_{1}(k+1)\right)-f\left(x_{1}(k)\right)\right\|<\delta .
\end{gathered}
$$

The controller design for this part is equivalent to the stabilization problem in the actuator saturation setting, which is solved in [9].

Step 2: Because the infinite zeros of $\Sigma$ are of order at most one, we can decompose the second subsystem as follows:

$$
\left\{\begin{aligned}
x_{d}(k+1) & =u_{1}(k)+G_{1} x_{1}(k)+G_{2} x_{c}(k)+E_{c} x_{d}(k) \\
x_{c}(k+1) & =A_{s+1} x_{c}(k)+E_{s+1} z(k)+ \\
& B_{2, s+1}\left(u_{3}(k)+G_{3} x_{1}(k)\right) \\
z_{1}(k)= & x_{d}(k) \\
z_{2}(k)= & u_{2}(k)
\end{aligned}\right.
$$

where we have decomposed

$$
u=\left(\begin{array}{l}
u_{1} \\
u_{2} \\
u_{3}
\end{array}\right), \quad z=\left(\begin{array}{l}
z_{1} \\
z_{2}
\end{array}\right), \quad x_{2}=\left(\begin{array}{l}
x_{c} \\
x_{d}
\end{array}\right),
$$

We can decompose $\&$ and $\mathcal{T}$ as

$$
s=s_{1} \times s_{2} \text {, and } \mathcal{T}=\mathcal{T}_{1} \times \mathcal{T}_{2}
$$

Recall that our initial conditions are such that that there exists $\rho>0$ such that:

$$
z(0) \in(1-\rho) \&, \quad z(1)-z(0) \in(1-\rho) \mathcal{T}
$$

Next we choose $\lambda$ such that:

$$
(1-\lambda) \& \subset \frac{\mathcal{T}}{4}
$$

$\lambda$ is well defined since without loss of generality we can assume $\delta$ is bounded. Partition $z_{0}(k)$ as

$$
z_{0}(k)=f\left(x_{1}(k)\right)=\left(\begin{array}{l}
f_{1}\left(x_{1}(k)\right) \\
f_{2}\left(x_{1}(k)\right)
\end{array}\right)
$$

Choose

$$
\begin{aligned}
& u_{2}=f_{2}\left(x_{1}(k)\right) \\
& u_{1}=\lambda\left(x_{d}(k)-f_{1}\left(x_{1}(k)\right)\right)+f_{1}\left(x_{1}(k+1)\right)-G_{1} x_{1}(k) \\
& -G_{2} x_{c}(k)-E_{c} x_{d}(k)
\end{aligned}
$$

Then we have:

$$
x_{d}(k+1)-f_{1}\left(x_{1}(k+1)\right)=\lambda\left(x_{d}(k)-f_{1}\left(x_{1}(k)\right)\right)
$$

which gives that:

$$
\begin{aligned}
x_{d}(k)=\lambda^{k} x_{d}(0)+ & \left(1-\lambda^{k}\right) f_{1}\left(x_{1}(k)\right)+ \\
& \lambda^{k}\left(f_{1}\left(x_{1}(k)\right)-f_{1}\left(x_{1}(0)\right)\right)
\end{aligned}
$$

It can be shown that for a given $\lambda \in[0,1]$ we can choose $\varepsilon$ and $\delta$ small enough to guarantee that this feedback satisfies $z(k) \in \delta$ and $z(k+1)-z(k) \in \mathcal{T}$ for all $k>0$. Moreover, $v=z-z_{0}$ satisfies (5.7) for a suitably chosen $M>0$. Finally we choose:

$$
u_{3}=F_{c} x_{c}-G_{3} x_{1}
$$

where $F_{c}$ is such that $A_{s+1}+B_{2, s+1} F_{c}$ is Schur-stable. Then it can be shown that the whole closed-loop system is asymptotically stable. 


\section{Non-right-invertible constraints}

For a system with non-right-invertible constraints, the $z$ equation in (5.1) can further be decomposed as

$$
\begin{gathered}
z=\left(\begin{array}{c}
z_{1} \\
z_{2}
\end{array}\right), \quad C_{z, 1}=\left(\begin{array}{c}
0 \\
C_{z, 12}
\end{array}\right), \\
C_{z, 2}=\left(\begin{array}{c}
C_{z, 21} \\
0
\end{array}\right), \quad D_{z}=\left(\begin{array}{c}
D_{z, 1} \\
0
\end{array}\right),
\end{gathered}
$$

We can isolate a subsystem which has input and output constraints:

$$
\Sigma_{1}:\left\{\begin{aligned}
x_{1}(k+1) & =A_{11} x_{1}(k)+K_{1} z_{1}(k) \\
z_{2}(k) & =C_{z, 12} x_{1}(k)
\end{aligned}\right.
$$

The remaining part of the system is strongly controllable and has no finite invariant zeros. Note that the constraint invariant zeros are precisely the unobservable eigenvalues of $\left(C_{z}, 12, A_{11}\right)$. In this case we have input and output constraints because both $z_{1}$ and $z_{2}$ are constrained. For semiglobal stabilization, this subsystem should have at most weakly non-minimum phase constraints. If this subsystem is still not right invertible, we have to continue decomposing it. In this way we obtain a chain of systems:

$$
\Sigma_{i}:\left\{\begin{aligned}
\tilde{x}_{i}(k+1) & =A_{i} \tilde{x}_{i}(k)+K_{i} \tilde{u}_{i}(k) \\
\tilde{z}_{i}(k) & =C_{z, i} \tilde{x}_{i}(k)
\end{aligned}\right.
$$

This chain ends if the final subsystem $\Sigma_{i}$ which is right invertible, Also possible is that after some steps $K_{i}=0$ which obviously implies that we can end the chain. It can be shown that if the pair $(A, B)$ of the given system $\Sigma_{d}$ is stabilizable, then all the systems $\Sigma_{i}$ as defined in (6.2) are stabilizable. The following theorem contains some necessary conditions for constrained global or semi-global stabilization when the system is not right invertible.

Theorem 6.1 Consider the system $\Sigma_{d}$ as given by (2.1). Let the sets $\&$ and $\mathcal{T}$ satisfy Assumption 2.1. Moreover, let the chain of systems $\Sigma_{i}(i=1, \ldots s)$ be as described above. Then the semi-global and global constrained stabilization problems formulated in Problem 2.3 and 2.4 are solvable only if the following conditions are satisfied:

(i) $(A, B)$ is stabilizable.

(ii) The constraint invariant zeros of the given system $\Sigma_{d}$ are all in the closed unit disc.

(iii) All the systems $\Sigma_{i}(i=1, \ldots s)$ have at most weakly non-minimum phase constraints.

(iv) The systems $\Sigma_{i}(i=1, \ldots s)$ with realization (6.2) satisfy:

$$
\operatorname{ker} C_{z, i} \subset \operatorname{ker} C_{z, i} A_{i}
$$

(v) The subsystem $\left(A, B, C_{z}, D_{z}\right)$ has no infinite zeros of order greater than one.

It can be shown that the condition in the above theorem is not sufficient.

Remark. Following the same arguments as in [5], we can develop a necessary and sufficient condition for solvability of constrained stabilization independent of the features of the given constraint sets. See [5] for details.

\section{Conclusions}

This paper has considered the semi-global and global stabilization problems of discrete-time linear systems in the presence of magnitude and rate constraints on both state and input variables. It turns out that the solvability conditions are largely dependent on the structural properties of linear plants such as the location of the constraint invariant zeros and the order of infinite zeros (or relative degree). The general results presented here include the stabilization problems of input constraints as a special case.

\section{References}

[1] D.S. Bernstein AND A.N. Michel, Guest Eds., Special Issue on saturating actuators, Int. J. Robust \& Nonlinear Control, 5(5), 1995.

[2] F. Blanchini, "Set invariance in control", Automatica, 35(11), 1999, pp. 1747-1769.

[3] E. Camacho And C. Bordons, Model predictive control, Springer Verlag, 1998.

[4] C.E. García, D.M. Prett, and M. Morari, "Model predictive control: theory and practice - a survey", Automatica, 25(3), 1989, pp. 335-348.

[5] A. Saberi, J. Han, ANd A.A. Stoorvogel, "Constrained stabilization problems for linear plants", Submitted for publication, 2000.

[6] P. SANnUti AND A. SABERI, "Special coordinate basis for multivariable linear systems - finite and infinite zero structure, squaring down and decoupling", Int. J. Contr., 45(5), 1987, pp. 1655-1704.

[7] E.D. SONTAG AND H.J. SUSSMANN, "Nonlinear output feedback design for linear systems with saturating controls", in Proc. 29th CDC, Honolulu, 1990, pp. 3414-3416.

[8] A. Saberi And A.A. SToorvogel, Guest Eds., Special Issue on control problems with constraints, Int. J. Robust \& Nonlinear Control, 9(10), 1999.

[9] A.A. Stoorvogel AND A. SABeri, "Output regulation of linear plants with actuators subject to amplitude and rate constraints", Int. J. Robust \& Nonlinear Control, 9(10), 1999, pp. 631-657. 